\title{
Low Complexity Burst Error Correcting Codes to Correct MBUs in SRAMs
}

\author{
Abhishek Das and Nur A. Touba \\ Computer Engineering Research Center, \\ University of Texas at Austin, TX-78712 \\ abhishekdas@utexas.edu, touba@ece.utexas.edu
}

\begin{abstract}
${ }^{1}$ Multiple bit upsets (MBUs) caused by high energy radiation is the most common source of soft errors in static random-access memories (SRAMs) affecting multiple cells. Burst error correcting Hamming codes have most commonly been used to correct MBUs in SRAM cell since they have low redundancy and low decoder latency. But with technology scaling, the number of bits being affected increases, thus requiring a need for increasing the burst size that can be corrected. However, this is a problem because it increases the number of syndromes exponentially thus increasing the decoder complexity exponentially as well. In this paper, a new burst error correcting code based on Hamming codes is proposed which allows much better scaling of decoder complexity as the burst size is increased. For larger burst sizes, it can provide significantly smaller and faster decoders than existing methods thus providing higher reliability at an affordable cost. Moreover, there is frequently no increase in the number of check bits or a very minimal increase in comparison with existing methods. A general construction and decoding methodology for the new codes is proposed. Experimental results are presented comparing the decoder complexity for the proposed codes with conventional burst error correcting Hamming codes demonstrating the significant improvements that can be achieved.
\end{abstract}

\section{KEYWORDS}

Burst error correction; Hamming codes; multiple bit upsets; SRAM; low complexity decoder

\section{ACM Reference Format:}

Abhishek Das and Nur A. Touba. 2018. Low Complexity Burst Error Correcting Codes to Correct MBUs in SRAMs. In GLSVLSI '18: 2018 Great Lakes Symposium on VLSI, May 23-25, 2018, Chicago, IL, USA. ACM, NY, NY, USA, 6 pages. https://doi.org/10.1145/3194554.3194570

\section{INTRODUCTION}

Soft errors caused by radiation poses a significant reliability

\footnotetext{
${ }^{1}$ Permission to make digital or hard copies of all or part of this work for personal or classroom use is granted without fee provided that copies are not made or distributed for profit or commercial advantage and that copies bear this notice and the full citation on the first page. Copyrights for components of this work owned by others than ACM must be honored. Abstracting with credit is permitted. To copy otherwise, or republish, to post on servers or to redistribute to lists, requires prior specific permission and/or a fee. Request permissions from Permissions@acm.org.

GLSVLSI '18, May 23-25, 2018, Chicago, IL, USA

(C) 2018 Association for Computing Machinery.

ACM ISBN 978-1-4503-5724-1/18/05 ..\$15.00

DOI: https://doi.org/10.1145/3194554.3194570
}

concern for SRAMs [4]. With technology scaling, the susceptibility of SRAMs to soft-errors has significantly increased as well [8]. In current nanoscale technology nodes, device geometries are small, and with technology scaling, devices are getting smaller. Thus, a particle strike might affect more than one cell causing a multiple bit upset [13]. The smaller the device geometries, more the number of cells that are affected by a single particle strike. A $b$-bit burst error caused by such a particle strike can cause multiple bits to be flipped within the $b$-bit burst window. Thus, codes aimed to correct MBUs in SRAMs should be able to correct all possible error combinations within a $b$-bit burst window including all $b$-bits getting flipped.

Burton Code [5] was one of the early codes that dealt with MBUs by correcting a single phased burst error or a single symbol error. But they can only correct $b$-bit burst error if it falls within a single symbol which is not always guaranteed. It is also possible to use multi-symbol error correction to correct all $b$-bit burst errors regardless of their position, but this increases the decoder complexity as well as the decoder latency. Over the years, many different and better codes have been devised to address the problem of correcting burst errors independent of their position. The most common method to address MBUs in SRAMs was to use a simple single error correcting double error detecting (SEC-DED) code in tandem with word-interleaving in such a way that each MBU would affect only a single bit for each word instead of multiple bits of a single word. This scheme made it possible to correct MBUs and reduce the soft error rate (SER) using a SEC-DED error correcting code (ECC) [3]. The degree of interleaving defined the amount of adjacent error protection for a memory system. However, such a scheme is no longer beneficial due to constraints such as memory aspect ratio, performance and power consumption which limits the degree of interleaving.

Error correcting codes (ECCs) with one-step majority logic decoding have been studied and extended to address the problem of MBUs for SRAMs. [7] proposed a general theory on adjacent error correcting orthogonal Latin square (OLS) codes for various burst sizes. [15] proposed a method to correct triple adjacent errors with the same amount of redundancy as a double error correcting OLS code. [14] proposed a method to correct MBUs in SRAMs using majority logic-decodable difference set codes. [16] proposed a new class for double adjacent error correction (DAEC) SEC-DED-DAEC codes from OLS codes. Although these classes of majority-logic decodable (MLD) codes have very low access latencies which is good for the overall performance of the memory system, the MLD codes suffer from very high redundancy. Other low redundancy codes have also been extensively studied for correcting MBUs in SRAMs. [11] proposed a parallel double error correcting Bose-ChaudhuriHocquenghem $(\mathrm{BCH})$ code. [10] proposed a single-bit and double 
adjacent error correcting parallel decoder for $\mathrm{BCH}$ codes. But such codes have very high decoder area and decoder latency. Multidimensional codes have also been proposed in the literature specifically to correct MBUs in SRAMs. [9] proposed a twodimensional coding method to correct multiple bit errors in caches. [2] proposed a matrix-based code which combined Hamming codes and Parity codes to detect and correct multiple errors. But both these codes have high area and power consumption compared to general burst error correcting Hamming codes. Recently, Hamming codes have also been studied and extended to correct MBUs in SRAMs. These codes provide balanced trade-off between the amount of redundancy required and the decoding complexity and decoding latency. Thus, they are an attractive solution specific to SRAMs. [6] first extended the traditional SEC-DED Hamming code to correct two adjacent errors for tolerating multiple bit upsets in small memories. [17] proposed a general solution for burst (local) error correcting codes working in conjunction with random (global) error correcting codes to correct MBUs in SRAMs. [12] presented a code with the basic SEC-DED coverage as well as both DAEC and scalable adjacent error detection ( $x \mathrm{AED}$ ) while reducing the adjacent/nonadjacent double-bit error mis-correction probability. [1] proposed a SEC-DAEC-TAEC and 3-bit burst error correction codes which reduced the total number of ones in the parity check matrix to optimize the decoder complexity and decoder latency. For all such Hamming extended codes, the decoding procedure still relies on a syndrome based matching method. As the burst size increases, the number of syndromes also increases exponentially, thus increasing the decoder complexity exponentially as well.

In this paper, a general $b$-bit burst error correcting code is proposed which aims to reduce the decoder complexity for a similar amount of redundancy as general burst error correcting Hamming codes. The proposed codes correct all possible combination of errors within a $b$-bit burst and achieve much better decoder complexity and decoder latency for higher burst sizes. The rest of the paper is organized as follows. Section 2 briefly describes the concepts for burst error correcting Hamming codes along with an analysis on syndromes. Section 3 describes the proposed codes, its construction and its decoding procedures. Section 4 evaluates the proposed codes and makes a comparison to the general burst error correcting Hamming codes. Finally, sec. 5 presents the conclusion of this work.

\section{BURST ERROR CORRECTING HAMMING CODES}

Extensive research has been done on burst error correcting Hamming codes as discussed previously. The key concepts related to burst error correcting codes remains the same. For a Hamming code to be $b$-bit burst error correcting, it needs to satisfy the following conditions [17]:

1. Each column of the parity check matrix is unique.

2. The bitwise XOR of any combination of columns within $b$ adjacent columns is unique.

Condition-1 ensures that all single errors are recognized and corrected. Condition-2 ensures that not only adjacent errors, but any combination of errors within the burst size $b$ are recognized and corrected. The parity check matrix can be constructed in an algorithmic manner by extending [6] or using a Boolean SAT solver [17]. An example of a parity check matrix for a 3-bit burst error correcting code for information length $k=16$ bits and with redundancy $r=8$ bits has been shown in Figure 1 .

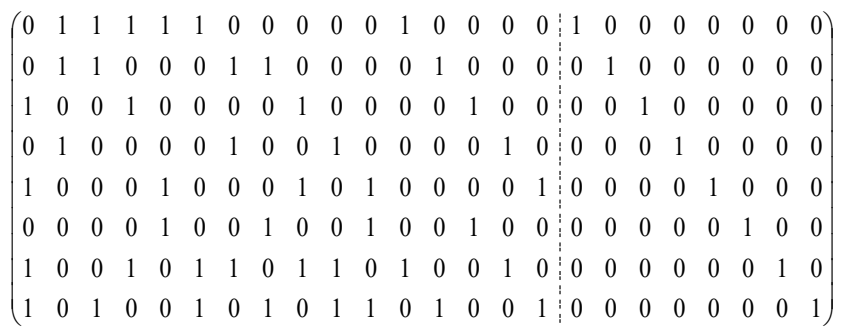

Figure 1: Parity check matrix of a 3-bit burst error correcting code for $k=16$.

\subsection{Syndrome Analysis}

The total number of syndromes for a $b$-bit burst error correcting code for each $b$-adjacent column code is given by equation (1). Only syndromes affecting information bits are taken into account as there is no necessary requirement to correct the parity check bits. Since for each information bit a $b$-bit burst might exist, the total number of useful syndromes for a $b$-bit burst error correcting code is given by equation (2), where $k$ is the total number of information bits.

$$
\begin{gathered}
\text { \# Syndromes } / \text { Burst }={ }^{b} C_{1}+{ }^{b} C_{2}+\cdots+{ }^{b} C_{b}=2^{b}-1 \\
\# \text { Syndromes }=k\left(2^{b}-1\right)
\end{gathered}
$$

As can be seen from equation (2), the total number of syndromes is a linear function of the length of information bits, and an exponential function of the burst size $b$. Thus, as the burst size increases the number of syndromes increase exponentially thereby increasing the decoder hardware exponentially. For a $b$-bit burst error, any combination of errors within $b$-adjacent bits is possible. Table I shows all the possible errors that can occur involving data bit $d_{i}$ within a 4-bit burst window. For each type of error within $b$ adjacent bits, there is a unique syndrome which defines the pattern and location of the error.

Table I: Possible Errors for a Data Bit Within a 4-Bit Burst Window

\begin{tabular}{|l|l|}
\hline $\begin{array}{l}\text { Number of } \\
\text { bits in error }\end{array}$ & Location of errors \\
\hline 1 & $i$ \\
\hline 2 & $(i, i+1) ;(i, i+2) ;(i, i+3) ;(i-3, i) ;(i-2, i) ;(i-1, i)$ \\
\hline \multirow{3}{*}{3} & $\begin{array}{l}(i-3, i-2, i) ;(i-3, i-1, i) ;(i-2, i-1, i) ;(i-2, i, i+1) ; \\
(i-1, i, i+1) ;(i, i+1, i+2) ;(i-1, i, i+2) ;(i, i+1, i+3) ; \\
(i, i+2, i+3)\end{array}$ \\
\hline 4 & $\begin{array}{l}(i-3, i-2, i-1, i) ;(i-2, i-1, i, i+1) ;(i-1, i, i+1, i+2) ; \\
(i, i+1, i+2, i+3)\end{array}$ \\
\hline
\end{tabular}

\subsection{Decoding Procedure}

The decoding procedure for most methods is based on syndrome matching i.e. each syndrome is mapped to particular data bit(s) being in error. Each syndrome is mapped to their corresponding data bits and the relevant syndrome is the $\mathrm{OR}$ of all corresponding 
syndromes. This indicates whether a bit is in error or not, and if it is in error, the data bit is flipped. Thus, as the burst size or the block length increases, the number of syndromes increases and so does the complexity of the decoding circuit.

\section{PROPOSED CODES}

For a burst size of $b$, the key idea of the proposed codes is to partition the parity check matrix in a manner such that the upper $b$ rows of the parity check matrix is used to compute the error pattern within the $b$-bit burst, and the lower $(r-b)$ rows of the parity check matrix are used to compute the location of the burst. To compute the error pattern, the upper $b$-rows of the parity check matrix are organized in such a manner that for each consecutive $b$-columns there is exactly one non-zero entry per row. This is easily done by interleaving the 1 s every $b$-columns. Next, the lower $(r-b)$ rows of the parity check matrix are constructed in such a manner that they satisfy the conditions for a $b$-bit burst error correcting Hamming Code:

1. All columns of the parity check matrix are unique.

2. The bitwise XOR of any combination of columns within $b$ adjacent columns are also unique.

Similar to burst error correcting Hamming codes, Condition-1 ensures that all single errors are recognized and corrected. Condition-2 ensures that not only adjacent errors, but any combination of errors within the burst size $b$ are recognized and corrected. This condition is necessary because MBUs do not necessarily flip consecutive bits, and there might be cases were the MBU flips a few non-consecutive bits within a burst size. The general form of the parity check matrix for a $b$-bit burst error correcting code has been shown in Figure 2. In this case $H^{*}$ refers to the lower $(r-b)$ rows constructed as described previously. The parity check matrix for a 4-bit burst error correcting code has been shown in Figure 3.

$$
H=\left(\begin{array}{ccccc}
I_{b} & I_{b} & \cdots & I_{b} & I_{r}
\end{array}\right)
$$

\section{Figure 2: General form of the parity check matrix of} proposed codes.

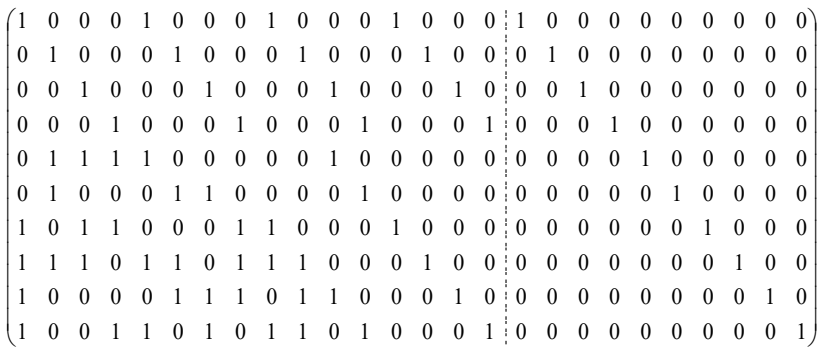

Figure 3: Parity check matrix of a 4-bit burst error correcting proposed codes for $k=16$.

The proposed codes are also systematic by design. Thus, the encoding procedure is the same as general Hamming codes. The parity bits are computed by XORing data-bits based on the parity check matrix and are appended to the data-bits to form the final codeword. As a result, the proposed codes do not add any complexity to the encoding procedure.

\subsection{Decoding Procedure}

The decoding procedure involves two parts: the error pattern computation and the error location computation. The error pattern is directly computed from the upper $b$-rows of the parity check matrix. Since the upper $b$-rows of the parity check matrix are arranged in an interleaved fashion, any error within a $b$-bit burst produces a syndrome equal to that of the error pattern. The location of the burst error is computed through the lower $(r-b)$ rows. If an error occurs within a $b$-bit burst starting from location $i$, the syndrome is given by equation (3).

Thus, the decoding works by considering each group of $b$ adjacent columns as a single symbol and decoding on a per symbol basis. Any data bit $d_{i}$ then is part of $b$ symbols of $b$-bit each. An example has been shown for a 4-bit burst error correcting code in Figure 4, the data but $d_{i}$ is part of 4-bit symbols $B_{i-3}, B_{i-2}, B_{i-1}$ and $B_{i}$. A $b$-bit burst error simply means an error in one of the $b$-bit symbols, and thus can be computed through equation (4). If for any of the $b$-bit symbols that the data bit is a part of equation (4) is satisfied, that means the data bit is in error. The error pattern of the data bit is simply the syndrome value $S_{\alpha}$ where $\alpha$ is the row amongst the upper $b$-rows for which the corresponding column is a 1 . Thus, the location of error for any data bit is the AND of all $b$-bit symbols of which it is a part. A data bit will not be in error only if it does not satisfy equation (4) for all $b$-bit symbols of which it is a part.

$$
\begin{gathered}
{\left[\begin{array}{c}
S_{1} \\
S_{2} \\
\vdots \\
S_{b} \\
S_{b+1} \\
S_{b+2} \\
\vdots \\
S_{r}
\end{array}\right]=\left[\begin{array}{c}
e_{i} \\
e_{i+1} \\
\vdots \\
e_{i+b-1} \\
h_{b+1, i} e_{i} \oplus h_{b+1, i+1} e_{i+1} \cdots \oplus h_{b+1, i+b-1} e_{i+b-1} \\
h_{b+2, i} e_{i} \oplus h_{b+2, i+1} e_{i+1} \cdots \oplus h_{b+2, i+b-1} e_{i+b-1} \\
\vdots \\
h_{r, i} e_{i} \oplus h_{r, i+1} e_{i+1} \cdots \oplus h_{r, i+b-1} e_{i+b-1}
\end{array}\right]} \\
h_{b+\beta, i} S_{1} \oplus h_{b+\beta, i+1} S_{2} \cdots \oplus h_{b+\beta, i+b-1} S_{b} \oplus S_{b+\beta}=0 \\
\forall \beta \in\{1,2, \ldots r-b\}
\end{gathered}
$$

The above method has a clear advantage over syndrome matching based decoding methods specifically for larger burst sizes. This is because as the burst size increases, the number of syndrome increases exponentially, as shown in equation (2). Thus, the amount of hardware needed to decode also increases exponentially in syndrome matching based decoding. But in the proposed decoding scheme, an increase in burst size results in addition of another term in equation (4). This essentially means a linear increase in the amount of XOR, AND and OR gates in the decoder design. A partial error pattern generator design for a 3-bit burst error correcting code for $k=16$ is shown in Figure 5. Similarly, the partial error pattern generator design for a 4-bit burst error correcting code for $k=16$ is shown in Figure 6. From Figure 5 and Figure 6, it can be seen that an increase in the burst size adds only a few XOR gates, an extra input to the AND gate and depending on the lower $(r-b)$ rows, possibly an extra input to the OR gate per symbol $B_{\alpha} \forall \alpha \in\{1, \ldots, k\}$. 


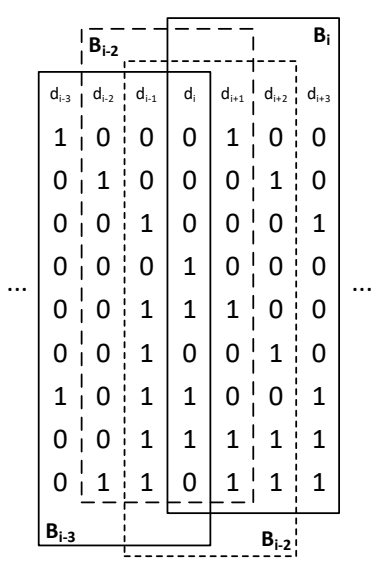

Figure 4: Symbols data bit $d_{i}$ is part of for a 4-bit burst error correcting proposed code.

\subsection{Area and Delay Optimization}

In a general Hamming Code, there are two optimization criteria that can be used on its parity check matrix to reduce the decoder complexity and decoder latency:

1. Minimize the total number of 1's in each row of the parity check matrix.

2. Minimize the total number of 1's in the parity check matrix.

The first criterion minimizes the decoder latency while the second criterion reduces the decoder complexity. A similar optimization can be done for the proposed codes as well but with a simple modification. Considering the general parity check matrix of the proposed codes shown in Figure 2, it can be seen that the upper $b$-rows of the matrix is already sparse and has the minimum number of 1's both on a row basis and in total. Thus, the two optimization criteria of the Hamming code can be applied to the lower $(r-b)$ rows of the matrix to achieve the same optimizations. This is because, minimizing the number of 1's in each row simply reduces the number of bits that need to be XORed together for the syndrome as well as for equation (4). Thus, the optimization results in lesser XOR operations thereby reducing the decoder delay. And minimizing the total number of 1's in the lower $(r-b)$ rows of the parity check matrix reduces the total number of XOR gates thus reducing the complexity. Thus, optimization techniques proposed in [1] are orthogonal to the proposed codes and can be used in place of burst error correcting Hamming codes.

\section{EVALUATION}

The burst error correcting codes for different burst sizes were constructed by extending the codes proposed in [17]. The proposed codes and the general burst error correcting Hamming codes were synthesized on Synopsys Design Compiler using NCSU FreePDK45 $45 \mathrm{~nm}$ library for information length $k=16,32$ and 64 bits, and burst sizes $b=3,4,5,6$ and 7 bits. For the general burst error correcting Hamming codes, the codes in [17] were used to construct the parity check matrix for difference burst sizes. But [17] doesn't explicitly define a decoding procedure and simply mentions the decoding

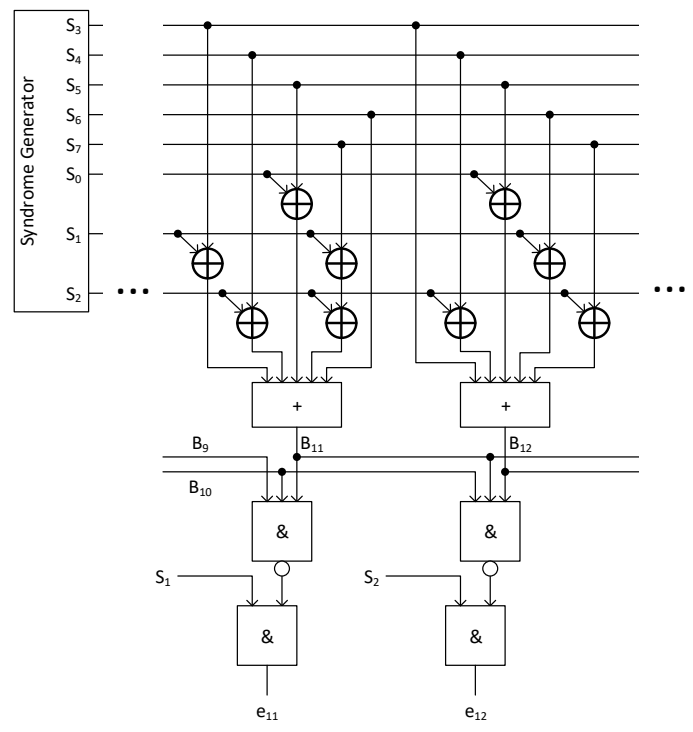

Figure 5: Partial schematics of the error pattern generator for a 3-bit burst error correcting proposed codes for $k=16$.

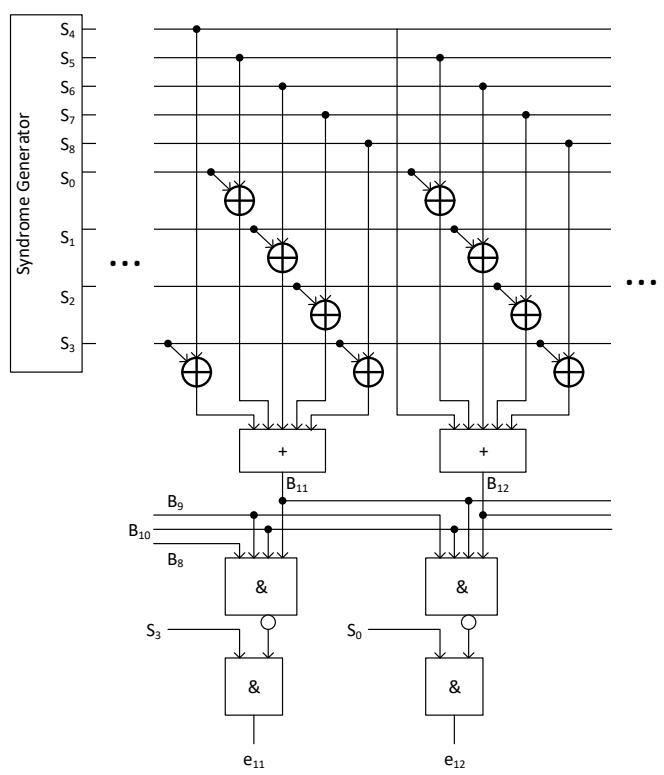

Figure 6: Partial schematics of the error pattern generator for a 4-bit burst error correcting proposed codes for $k=16$.

procedure to be the same as a Hamming Code. Thus, the syndrome matching based decoding from [6] was extended to the appropriate burst sizes for the constructed parity check matrix derived from [17]. The proposed codes and the general burst error correcting Hamming codes were implemented using Dataflow model in Verilog and errors were injected to ensure that all errors within the burst size was corrected. Exhaustive testing was done for all different error patterns within the burst size and for various locations of the burst errors. Table II shows the comparison of redundancy, decoder 
Table II: Comparison of redundancy, decoder latency, decoder area and decoder cell usage between traditional Hamming code and Proposed Codes.

\begin{tabular}{|c|c|c|c|c|c|c|c|c|c|}
\hline \multirow{2}{*}{$\begin{array}{l}\text { \#Data bits } \\
\text { (k) }\end{array}$} & \multirow{2}{*}{$\begin{array}{c}\text { Burst error size } \\
\text { (b-bits) }\end{array}$} & \multicolumn{4}{|c|}{ Burst error correcting Hamming Codes } & \multicolumn{4}{|c|}{ Proposed Codes } \\
\hline & & \#check bits (r) & Latency (ns) & \#cells & $\operatorname{Area}\left(\mu m^{2}\right)$ & \#check bits (r) & Latency (ns) & \#cells & $\operatorname{Area}\left(\mu m^{2}\right)$ \\
\hline \multirow{5}{*}{16} & 3 & 8 & 0.89 & 302 & 807 & 8 & 0.79 & 225 & 892 \\
\hline & 4 & 9 & 1 & 531 & 1327 & 9 & 0.93 & 252 & 977 \\
\hline & 5 & 11 & 1.31 & 1015 & 2396 & 12 & 0.9 & 269 & 963 \\
\hline & 6 & 13 & 1.33 & 1559 & 3570 & 13 & 1.23 & 339 & 1162 \\
\hline & 7 & 15 & 1.5 & 2795 & 6360 & 15 & 1.16 & 276 & 921 \\
\hline \multirow{5}{*}{32} & 3 & 8 & 1.11 & 529 & 1480 & 9 & 1.08 & 450 & 1711 \\
\hline & 4 & 10 & 1.29 & 1108 & 2759 & 10 & 1.15 & 506 & 2020 \\
\hline & 5 & 12 & 1.59 & 2115 & 4983 & 12 & 1.29 & 674 & 2708 \\
\hline & 6 & 14 & 1.9 & 4354 & 9915 & 14 & 1.4 & 727 & 2965 \\
\hline & 7 & 15 & 2.39 & 7753 & 17482 & 16 & 1.27 & 650 & 2493 \\
\hline \multirow{5}{*}{64} & 3 & 9 & 1.47 & 1056 & 2967 & 10 & 1.18 & 858 & 3150 \\
\hline & 4 & 11 & 1.89 & 2032 & 5171 & 11 & 1.22 & 1016 & 3657 \\
\hline & 5 & 13 & 1.8 & 4102 & 9696 & 13 & 1.4 & 1270 & 4774 \\
\hline & 6 & 14 & 2.51 & 8952 & 20296 & 14 & 1.58 & 1724 & 7022 \\
\hline & 7 & 16 & 2.52 & 15958 & 36124 & 16 & 1.77 & 1748 & 7495 \\
\hline
\end{tabular}

area, number of cells in decoder and decoder latency, between the proposed codes and the general burst error correcting Hamming codes, for different information lengths and different burst error sizes. The number of information bits in a codeword is given by $k$. The burst size $b$ refers to the maximum number of bits in a single burst that the code can correct. The redundancy is given by the total number of check-bits $(r)$ required to correct a single burst error for the given number of information bits. The decoder latency is the longest timing path between the codeword and the decoded message bits.

\subsection{Redundancy}

Table II shows the comparison of redundancy for $k=16,32$ and 64 bits for various burst error sizes $b=3,4,5,6$ and 7 bits. The redundancy column shows the number of check bits required to correct the $b$-bit burst error for different information lengths $k$. It can be seen that the redundancy in all the cases is either the same or very close to the redundancy of the general burst error correcting Hamming codes. Thus, the proposed codes add negligible amount of redundancy compared to the general burst error correcting Hamming codes.

\subsection{Hardware Complexity}

Table II shows the comparison of decoder latency, number of cells as well as decoder area for $k=16,32$ and 64 bits for various burst error sizes $b=3,4,5,6$ and 7 bits. It can be seen that the decoder area for general burst error correcting Hamming codes increases almost exponentially with the increase in burst size. This in turn affects the decoder latency as well since the gate depth also increases with the increase in burst size. The decoder area for the proposed codes increases almost linearly with an increase in burst size as shown in Figure 7, which plots the decoder area for both the general burst error correcting Hamming codes and the proposed codes for different information lengths $k$ and different burst error sizes $b$. The decoder area is much less for the proposed codes compared to the general burst error correcting Hamming codes for higher burst sizes. This also leads to a slower increase in decoder latency compared to the general burst error correcting Hamming codes. Figure 8 plots the decoder latency for the general burst error correcting Hamming codes and the proposed codes for different information lengths $k$ and different burst error sizes $b$. Thus, the decoder latency is also lower for higher burst sizes compared to the general burst error correcting Hamming codes. It is also seen that in the cases of $k=16$ and 32, the decoder complexity and the decoder latency for burst size $b=7$ is lower compared to burst size $b=5,6$. This is because the H-matrix for the proposed codes is constructed using a greedy algorithm, that selects the lowest Hamming weight column that satisfies the conditions described in Sec. 3. This inadvertently creates a very sparse H-matrix, i.e. it has lesser total number of $1 \mathrm{~s}$, in some cases compared to other cases. The constructed H-matrix from our algorithm is sparser for burst size $b=$ 7 compared to the H-matrix of burst size $b=5$, 6, which in turn reduces the decoder complexity for burst size $b=7$.

\section{CONCLUSION}

This paper proposes a new burst error correcting code by modifying the parity check matrix of a general burst error correcting Hamming code along with its decoding procedure. The proposed codes achieve significant reduction in decoder area as well as a nonnegligible reduction in the decoder latency for similar amount of 


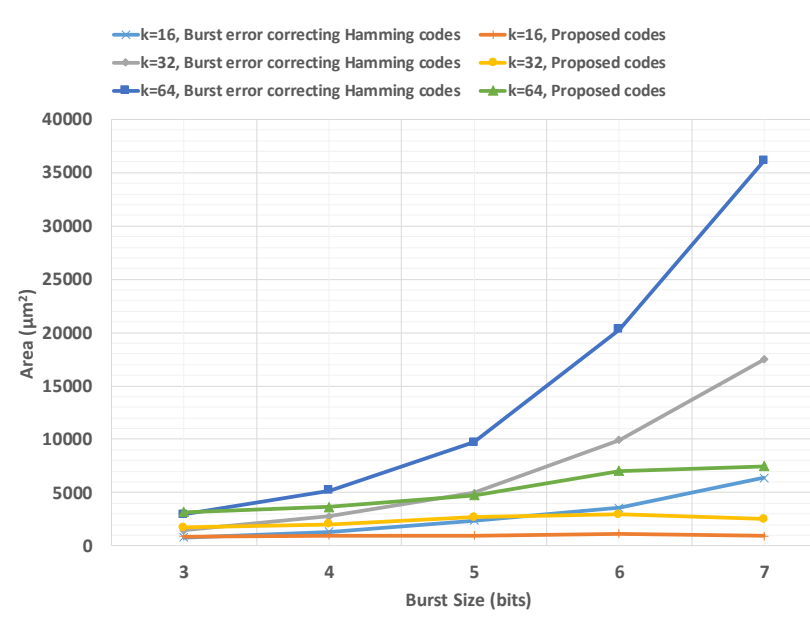

Figure 7: Comparison of decoder area for different information lengths $k$ and different burst sizes $b$.

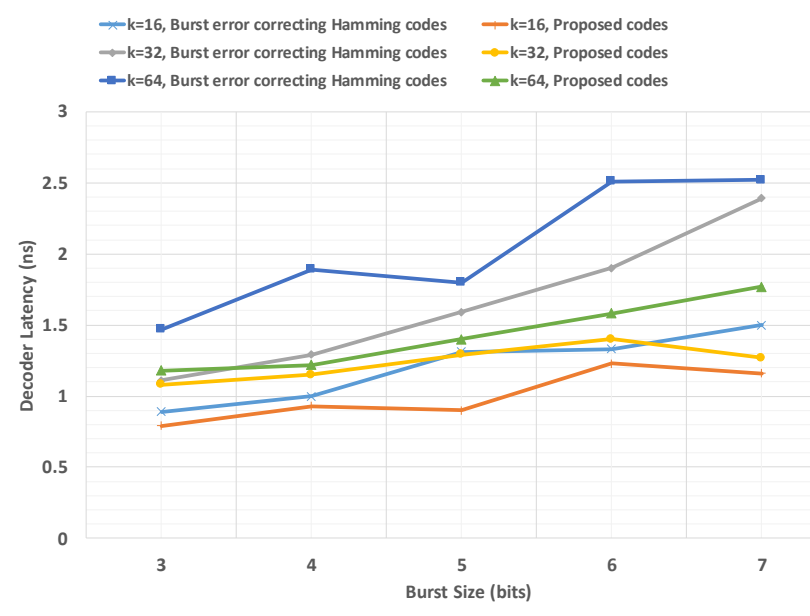

Figure 8: Comparison of decoder latency for different information lengths $k$ and different burst sizes $b$.

redundancy compared to a general burst error correcting Hamming Code. Also, the proposed codes can be used in tandem with other burst error correction optimization methods to achieve better decoder latency as well as better decoder complexity. Thus, the proposed codes are highly suitable for correcting MBUs in SRAMs specifically for lower technology nodes where the burst size from a single particle strike is expected to increase.

\section{ACKNOWLEDGMENTS}

This research was supported in part by the National Science Foundation under Grant No. CCF-1617665.

\section{REFERENCES}

[1] L. S. Adalid, P. Reviriego, P. Gil, S. Pontarelli and J. A. Maestro, "MCU Tolerance in SRAMs Through Low-Redundancy Triple Adjacent Error Correction," in IEEE Transactions on Very Large Scale Integration (VLSI) Systems, vol. 23, no. 10, pp. 2332-2336, Oct. 2015.
[2] C. Argyrides, D. Pradhan and T. Kocak, "Matrix codes for reliable and cost efficient memory chips," in IEEE Transactions on Very Large Scale Integration (VLSI) Systems, vol. 19, no. 3, pp. 420-428, Mar. 2011

[3] S. Baeg, S. Wen and R. Wong, "SRAM Interleaving Distance Selection with a Soft Error Failure Model," in IEEE Transactions on Nuclear Science, vol. 56, no. 4, pp. 2111-2118, Aug. 2009.

[4] R. Baumann, "Soft errors in advanced computer systems," in IEEE Design \& Test of Computers, vol. 22, no. 3, pp. 258-266, May-Jun. 2005.

[5] H. O. Burton, "Some asymptotically optimal burst-correction codes and their relation to single-error-correcting reed-solom codes," in IEEE Transactions on Information Theory, vol. 17, no. 1, pp. 92-95, Jan. 1971.

[6] A. Dutta and N.A. Touba "Multiple Bit Upset Tolerant Memory Using a Selective Cycle Avoidance Based SEC-DED-DAEC Code," in Proc. of IEEE VLSI Test Symposium, pp. 349-354, 2007.

[7] R. Datta and N.A. Touba, "Generating Burst-Error Correcting Codes from Orthogonal Latin Square Codes - A Graph Theoretic Approach," in Proc. of IEEE Symposium on Defect and Fault Tolerance, pp. 367-373, 2011.

[8] E. Ibe, H. Taniguchi, Y. Yahagi, K. Shimbo and T. Toba, "Impact of scaling on neutron-induced soft error in SRAMs from a $250 \mathrm{~mm}$ to a 22 $\mathrm{nm}$ design rule," in IEEE Transactions on Electron Devices, vol. 57, no. 7, pp. 1527-1538, Jul. 2010

[9] J. Kim, N. Hardavellas, K. Mai, B. Falsafi and J. Hoe, "Multi-bit error tolerant caches using two-dimensional error coding," in Annual IEEE/ACM International Symposium on Microarchitecture (MICRO), pp. 197-209, 2007.

[10] K. Namba, S. Pontarelli, M. Ottavi and F. Lombardi, "A Single-Bit and Double-Adjacent Error Correcting Parallel Decoder for Multiple-Bit Error Correcting BCH Codes," in IEEE Transactions on Device and Materials Reliability, vol. 14, no. 2, pp. 664-671, Jun. 2014.

[11] R. Naseer and J. Draper, "Parallel double error correcting code design to mitigate multi-bit upsets in SRAMs," in Proc. of Eurpoean Solid-State Circuits Conference (ESSCIRC), pp. 222-225, 2008.

[12] A. Neale and M. Sachdev, "A new SEC-DED error correction code subclass for adjacent MBU tolerance in embedded memory," in IEEE Transactions on Device and Materials Reliability, vol. 13, no. 1, pp. 223230, Mar. 2013

[13] D. Radaelli, H. Puchner, S. Wong and S. Daniel, "Investigation of multi-bit upsets in a $150 \mathrm{~nm}$ technology SRAM device," in IEEE Transactions on Nuclear Science, vol. 52, no. 6, pp. 2433-2437, Dec. 2005.

[14] P. Reviriego, M. Flanagan, S.-F. Liu and J. Maestro, "Multiple cell upset correction in memories using difference set codes," in IEEE Transactions on Circuits and Systems-I: Regular Papers, vol. 59, no. 11, pp. 2592-2599, Nov. 2012.

[15] P. Reviriego, S. Liu, J.A. Maestro, S. Lee, N.A. Touba and R. Datta, "Implementing Triple Adjacent Error Correction in Double Error Correction Orthogonal Latin Square Codes," in Proc. of IEEE Symposium on Defect and Fault Tolerance, pp. 167-171, 2013.

[16] P. Reviriego, S. Pontarelli, A. Evans and J. A. Maestro, "A Class of SECDED-DAEC Codes Derived from Orthogonal Latin Square Codes", in IEEE Transactions on Very Large Scale Integration (VLSI) Systems, vol. 23, no. 5, pp. 968-972, May 2015.

[17] S. Shamshiri and K. T. Cheng, "Error-locality-aware linear coding to correct multi-bit upsets in SRAMs," in Proc. of IEEE International Test Conference (ITC), Paper 7.1, 2010. 